\title{
Bounded Labeling Function for Global Segmentation of Multi-Part Objects with Geometric Constraints
}

\author{
Masoud S. Nosrati, Shawn Andrews, Ghassan Hamarneh \\ Medical Image Analysis Lab, Simon Fraser University, BC, Canada \\ $\{$ smn6, sda56, hamarneh\}esfu.ca
}

\begin{abstract}
The inclusion of shape and appearance priors have proven useful for obtaining more accurate and plausible segmentations, especially for complex objects with multiple parts. In this paper, we augment the popular MumfordShah model to incorporate two important geometrical constraints, termed containment and detachment, between different regions with a specified minimum distance between their boundaries. Our method is able to handle multiple instances of multi-part objects defined by these geometrical constraints using a single labeling function while maintaining global optimality. We demonstrate the utility and advantages of these two constraints and show that the proposed convex continuous method is superior to other state-of-theart methods, including its discrete counterpart, in terms of memory usage, and metrication errors.
\end{abstract}

\section{Introduction}

The piecewise constant Mumford-Shah (MS) model [18] is one of the most popular models in image segmentation. In recent years, many efforts have been made to improve this model in terms of optimizability, by simplifying the objective function and formulating it as a convex energy functional [2, 7, 20], and fidelity, by making the objective function more faithful to the underlying segmentation tasks. In image segmentation literature, improving objective function fidelity has taken several forms: (i) adding new energy terms, e.g. edge, region, and, shape prior terms [1]; (ii) extending binary segmentation methods to multi-label segmentation [8, 26]; and (iii) incorporating spatial relationships between labels, objects, or object parts [14]. In many image labeling tasks, some geometric relationships are known beforehand, and incorporating this information into a segmentation algorithm improves results.

In this paper, we focus on segmentation tasks where two regions must be separated by a third. Specifically, we focus primarily on the geometric constraint containment,

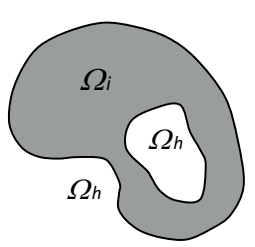

(a) Standard labeling function setting

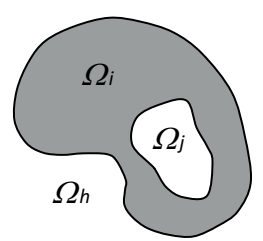

(b) Our setting
Figure 1: The inside vs. outside ambiguity in (a) is resolved by our containment constraint in (b).

where one region separates a second region from the background (cf. Fig. 1). Other geometric constraints can also be enforced using the same framework, such as detachment, where the background separates two regions. This paper addresses the problem of multi-region segmentation with these two important geometrical constraints, containment and detachment with a minimum distance (or thickness) between regions' boundaries, in a continuous framework while maintaining global optimality. We choose these two geometrical constraints due to their intuitive definitions, descriptive power, and ability to help properly segment regions with weak intensity/color appearance models. Using a continuous framework provides several advantages over discrete methods: 1) no metrication error; 2) less memory usage; 3) efficient parallelizability, and 4) allowance for sub-pixel resolution.

\subsection{Previous works}

Improving segmentation via encoding spatial relations between multiple target objects is not new. For example, some methods encode spatial relationships via relative interobject distances [17] while other works have incorporated fuzzy spatial relationships [9], but those methods do not handle stricter geometric constraints such as containment. Another popular approach is to perform atlas-based segmentation, which has been particularly useful in medical image analysis applications since an atlas encodes the spatial relationships between multiple anatomical structures, or 


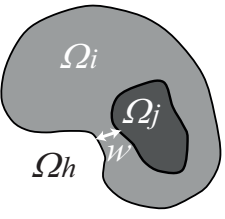

(a)

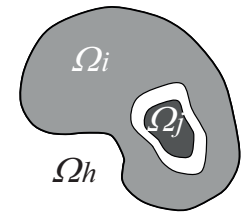

(b)

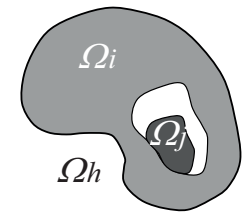

(c)
Figure 2: Containment vs. similar configurations ( $h$ : background). According to (3), "object $i$ contains object $j$ " in (a) with $T h\left(\Omega_{h}, \Omega_{i}, \Omega_{j}\right) \geq w$, but the relationship between $i$ and $j$ in (b) and (c) is not containment.

organs [10]. However, those methods have been designed to encode constraints on a single instance of an object in an image, not multiple instances, spatially-recurring throughout the image.

A few recent works have focused on tiered segmentation to encode adjacency relationships [12,21]. Strekalovskiy et al. [21] proposed a generalized label ordering constraint which can enforce many complex geometric constraints while maintaining convexity. This method requires that the constraint term obey the triangle inequality, a requirement that was later relaxed by introducing a convex relaxation method for non-metric priors [22]. Both [21] and [22] are designed to penalize transitions between adjacent labels, but for meaningful containment and detachment constraints, a minimum spatial distance between non-adjacent labels is required. Other recent works have focused on incorporating topological constraints into a segmentation objective function. In the continuous domain, some methods incorporate the containment constraint into their segmentation framework by simultaneously evolving a coupled surfaces [19, 23, 25, 28]. However, these works are limited to objects with two surfaces and also are limited to segmenting a single instance of each object in an image. In the discrete domain, Li et al. [16] proposed a method to segment nested objects, but their method is limited to star-shaped objects. Delong and Boykov [11] and Ulén et al. [24] proposed segmentation methods that encode geometric constraints (including containment) between distinct regions into a graph cut framework. Our work can be viewed as a continuous analogue to these works, providing several advantages, as noted earlier and as will demonstrated in Section 4

We represent our segmentation using a single continuous labeling function, assigning each region to an interval of label values. We perform segmentation via energy minimization, and we ensure a globally optimal solution using a "functional lifting" technique, similar to what is used by Pock et al. [20], to convexify our data energy term by reformulating the problem in a higher dimensional space. This approach exhibits several important qualities, such as the ability to deal with topological changes (e.g. spatially recurring instances of an object), and extendibility to higher dimensional images.

We introduce the containment and detachment constraints in Section 2. We show how to encode these two constraints in a continuous segmentation framework and show how our formulation can be convexified by functional lifting in Section 2.1. Section 3 explains how the energy is optimized. Different examples as well as comparisons with other popular state-of-the-art methods are given in Section 4. followed by our conclusions in Section 5 .

\section{Methodology}

In this section we explicitly define containment and detachment and show how we encode them in a MS based model while maintaining global optimality.

We first consider a containment constraint in a 3 region segmentation. We divide the image domain, $\Omega \subset \mathbb{R}^{2}$, into three non-overlapping parts (Fig. 1. b)): the outside or background region $\Omega_{h}$, the outer region $\Omega_{i}$, and the contained region $\Omega_{j}$, where $\Omega=\Omega_{h} \cup \Omega_{i} \cup \Omega_{j}$. In many binary segmentation applications that use relaxed labeling functions, label values below $1 / 2$ correspond to background and values above $1 / 2$ correspond to foreground. We extend this definition as follows. Given a label set $\Gamma=[0,1]$, we define our labeling function $u: \Omega \rightarrow \Gamma$, such that

$$
\begin{aligned}
0 \leq u(\boldsymbol{x})<1 / 3 & \Longleftrightarrow \boldsymbol{x} \in \Omega_{h} \\
1 / 3 \leq u(\boldsymbol{x})<2 / 3 & \Longleftrightarrow \boldsymbol{x} \in \Omega_{i} \\
2 / 3 \leq u(\boldsymbol{x})<1 & \Longleftrightarrow \boldsymbol{x} \in \Omega_{j} .
\end{aligned}
$$

To precisely define containment, we introduce a function that measures the thickness of the outer region $\Omega_{i}$ :

$$
\operatorname{Th}\left(\Omega_{h}, \Omega_{i}, \Omega_{j}\right)=\min _{x_{1} \in \Omega_{j}} \min _{x_{2} \in \Omega_{h}}\left\|x_{1}-x_{2}\right\| .
$$

We define containment for 3 regions as:

Definition 1 (Containment). We say object $i$ contains object $j$ with thickness $w$ if and only if

$$
T h\left(\Omega_{h}, \Omega_{i}, \Omega_{j}\right) \geq w .
$$

We note that $\Omega=\Omega_{h} \cup \Omega_{i} \cup \Omega_{j}$ is assumed here.

An example is shown in Fig. 2(a) where the light gray object, $i$, contains the dark gray object, $j$, with a minimum thickness of $w$. The related configurations between $i$ and $j$ seen in Fig. 2(b) and (c) are not containment based on our definition in (3). However, (b) can be seen as containment in a 4 region segmentation: $i$ contains the interior white region, and the interior white region contains $j$.

Given an input image $1: \Omega \subset \mathbb{R}^{2} \rightarrow \mathbb{R}$, for objects $i$ and $j$ and the background $h$, let $\mu_{i}, \mu_{j}$ and $\mu_{h}$ be constant approximations of the regional intensities and define

\footnotetext{
${ }^{1}$ Our method can be extended to vector valued images, e.g. color images or tensor fields, by modifying the data terms, as in [6], |27]
} 
$g_{k}(\boldsymbol{x})=\left|I(\boldsymbol{x})-\mu_{k}\right|^{2}$ for $k=\{h, i, j\}$. To segment $I$ such that $i$ contains $j$ we solve the following energy minimization problem:

$$
\begin{gathered}
\underset{u \in D}{\arg \min } E(u, \mathbf{g}) \\
=\underset{u \in D}{\arg \min } \int_{\Omega}\left|\nabla_{\boldsymbol{x}} u(\boldsymbol{x})\right|+\rho(\boldsymbol{x}, u(\boldsymbol{x}), \mathbf{g}) d \boldsymbol{x}, \\
D=\{u \mid u(\boldsymbol{x})=0 \text { for } x \in \partial \Omega \text { and } \\
\left.\quad T h\left(\Omega_{h}, \Omega_{i}, \Omega_{j}\right) \geq w\right\} .
\end{gathered}
$$

Here $\nabla_{\boldsymbol{x}}$ is the gradient in $x$ and $y$ directions, $\mathbf{g}=$ $\left(g_{h}, g_{i}, g_{j}\right)$, and $\rho(\boldsymbol{x}, u(\boldsymbol{x}), \mathbf{g}): \Omega \rightarrow \mathbb{R}^{+}$is a non-negative data term that encourages $u$ to satisfy (1), e.g.

$$
\rho(\boldsymbol{x}, u(\boldsymbol{x}), \mathbf{g})=\left\{\begin{array}{ll}
g_{h}(\boldsymbol{x}) & \text { if } 0 \leq u(\boldsymbol{x})<1 / 3 \\
g_{i}(\boldsymbol{x}) & \text { if } 1 / 3 \leq u(\boldsymbol{x})<2 / 3 \\
g_{j}(\boldsymbol{x}) & \text { if } 2 / 3 \leq u(\boldsymbol{x})<1
\end{array} .\right.
$$

For convenience, we often let $\rho$ be a function of $\mathbf{g}$ implicitly, and write $\rho(\boldsymbol{x}, u(\boldsymbol{x}))$.

Constraining $u$ to $D, E(u, \mathbf{g})$ ensures that object $j$ and object $h$ have no shared boundaries, resulting in $j$ being contained in $i$. In other words, the segmentation corresponding to $u$ cannot abruptly change from object $j$ to object $h$, and thus the value of $u$ cannot change from $u \geq 2 / 3$ to $u \leq 1 / 3$ in a distance less than $w$.

This leads us to the fact that the constraint $T h\left(\Omega_{h}, \Omega_{i}, \Omega_{j}\right) \geq w$ can be replaced by the more convenient constraint $\left|\nabla_{\boldsymbol{x}} u\right| \leq \frac{1}{3 w}$, which limits the rate that $u$ can change spatially. This lets us rewrite $D$ as

$$
D=\left\{u \mid u(\boldsymbol{x})=0 \text { for } x \in \partial \Omega,\left|\nabla_{\boldsymbol{x}} u\right| \leq \frac{1}{3 w}\right\}
$$

To better understand the equivalence of these constraints, let's consider the example shown in Fig. 3 Here, a black and white image is segmented into three regions, with $\mu_{h}$ corresponding to black pixels, $\mu_{i}$ corresponding to (nonexistent) gray pixels, and $\mu_{j}$ corresponding to white pixels. Fig. 3. b) illustrates the labeling function $u$ corresponding to the segmentation in Fig. 3 (a), with no thickness constraint. Here, $u$ becomes discontinuous (unbounded $\left|\nabla_{\boldsymbol{x}} u\right|$ ) in order to avoid assigning any pixels to the exterior object $i$. By enforcing the restriction $\left|\nabla_{\boldsymbol{x}} u\right| \leq \frac{1}{3 w}, u$ is not able to jump from $<1 / 3$ (background) to $\geq 2 / 3$ (white object) in less than distance $w$ (Fig. 3(d)). By restricting $u$, we force the white object to be contained by an intermediate region of thickness of $w$ (cf. Fig. 3(c)). We note that if $w$ is large enough, the energy increase from hallucinating the intermediate region in Fig. 3.(c) will become greater than the energy increase from not segmenting the inner white region, and the result will be $u=0$ across the image.

Using a similar formulation to containment, we can incorporate a constraint ensuring two regions are detached.

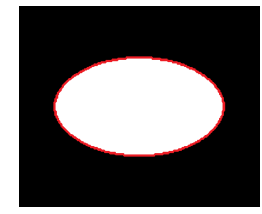

(a) Unconstrained

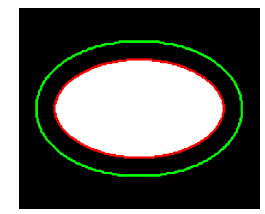

(c) Constrained segmentation segmentation

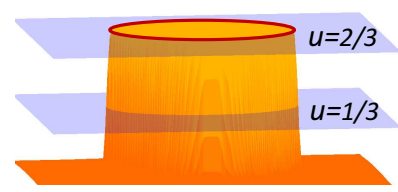

(b) $u$ corresponding to (a)

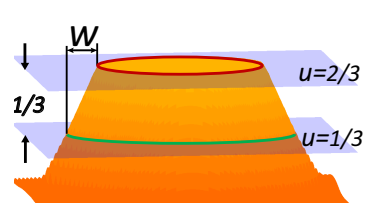

(d) $u$ corresponding to (c), $\left|\nabla_{\boldsymbol{x}} u\right| \leq 1 / 3 w$
Figure 3: Constrained vs. unconstrained labeling function $u$. In (a), the 3-region labeling function $u$, as defined in (1), is used to segment the white object from the black background, while the intermediate region has zero thickness. In (b), we see the $u$ corresponding to (a). Without a thickness constraint, $u$ is allowed to become discontinuous, skipping over the interval corresponding to the intermediate region, i.e. $\Omega_{i}$ in (1). In (c), we see how the segmentation changes when the thickness constraint 3 is enforced, with the intermediate region being hallucinated around the white object. In (d), we see the $u$ corresponding to (c). By bounding the rate of change of $u$, a band of thickness $w$ must be assigned to the intermediate region.

Definition 3 (Detachment). Object $i$ and object $j$ are detached with thickness $w$ if and only if

$$
\operatorname{Th}\left(\Omega_{i}, \Omega_{h}, \Omega_{j}\right) \geq w .
$$

In other words, object $j$ does not share a boundary with object $i$, thereby ensuring a separation between $i$ and $j$ by enforcing the labeling function $u$ to pass through the background, $h$, as it travels from $i$ to $j$. To encode detachment, we simply swap $g_{h}$ and $g_{i}$ in (4).

Now, we note that the data term (5) is not convex, making standard gradient descent based optimization schemes prone to local minima. In the next section, we discuss how to convexify (4) and thus find a globally minimizing segmentation.

\subsection{Function convexification}

We use a "functional lifting" technique similar to the one proposed by Pock et al. [20] (motivated by Ishikawa's work in the discrete Markov random field setting [15]) to transfer our energy functional to a higher dimensional space, where it becomes convex. The objective is to solve the following minimization problem

$$
\underset{u \in D}{\arg \min }\left\{\int_{\Omega}\left|\nabla_{\boldsymbol{x}} u(\boldsymbol{x})\right| d \boldsymbol{x}+\int_{\Omega} \rho(\boldsymbol{x}, u(\boldsymbol{x})) d \boldsymbol{x}\right\} .
$$


The first term is a convex total variation (TV) term, but the second term $\rho(\boldsymbol{x}, u(\boldsymbol{x}))$ can be non-convex. To lift the original energy to a higher dimensional space, we represent $u$ in terms of its super level set, $\varphi: \Omega \times \Gamma \rightarrow\{0,1\}$ by

$$
\varphi(\boldsymbol{x}, \gamma)=\mathbf{1}_{\{u \geq \gamma\}}(\boldsymbol{x})= \begin{cases}1 & \text { if } u(\boldsymbol{x}) \geq \gamma \\ 0 & \text { otherwise }\end{cases}
$$

$u$ can be recovered from $\varphi$ via the layer-cake formula

$$
u(\boldsymbol{x})=\int_{\Gamma} \varphi(\boldsymbol{x}, \gamma) d \gamma
$$

The TV term in (8) can be re-written with respect to $\varphi$ using the generalized co-area formula [13]:

$$
\int_{\Omega}\left|\nabla_{\boldsymbol{x}} u(\boldsymbol{x})\right| d \boldsymbol{x}=\int_{\Omega} \int_{\Gamma}\left|\nabla_{\boldsymbol{x}} \varphi(\boldsymbol{x}, \gamma)\right| d \gamma d \boldsymbol{x}
$$

By observing that $\delta(u(\boldsymbol{x})-\gamma) \equiv\left|\partial_{\gamma} \varphi(\boldsymbol{x}, \gamma)\right|^{2}$, where $\delta(\cdot)$ is the Dirac delta function, the data term in 80 can then be re-written as [20]:

$$
\begin{aligned}
\rho(\boldsymbol{x}, u(\boldsymbol{x})) & =\int_{\Gamma} \rho(\boldsymbol{x}, \gamma) \delta(u(\boldsymbol{x})-\gamma) d \gamma \\
& =\int_{\Gamma} \rho(\boldsymbol{x}, \gamma)\left|\partial_{\gamma} \phi(\boldsymbol{x}, \gamma)\right| d \gamma
\end{aligned}
$$

Now, using (11) and (12), the equivalent form of (8) is

$$
\underset{\{\varphi \mid u \in D\}}{\arg \min } \int_{\Omega} \int_{\Gamma}\left|\nabla_{\boldsymbol{x}} \varphi(\boldsymbol{x}, \gamma)\right|+\rho(\boldsymbol{x}, \gamma)\left|\partial_{\gamma} \varphi(\boldsymbol{x}, \gamma)\right| d \gamma d \boldsymbol{x}
$$

Note that the non-convex function $\rho$ does not depend on $\varphi$ any more and 13 is convex in $\varphi$. As the last stage of convexification, $\varphi, 9$, is relaxed so it varies continuously between zero and one, i.e. $\varphi \in \Omega \times \Gamma \rightarrow[0,1]$. To recover $u$, we threshold $\varphi$ and apply 10 .

Now, it is not immediately clear what form the constraint $\{\varphi \mid u \in D\}$ will take. If we ignore the thickness constraint from $D$, we could use $\varphi \in \mathcal{D}_{1}$ where

$$
\mathcal{D}_{1}=\left\{\varphi \mid \varphi(\boldsymbol{x}, 0)=1, \varphi(\boldsymbol{x}, 1)=0, \partial_{\gamma} \varphi \leq 0\right\} .
$$

It is evident from (9) that every $\varphi$ constructed from the super level sets of some $u$ are in $\mathcal{D}_{1}$.

We now present a theorem describing how to enforce the thickness constraint in $\varphi$. Let $\nabla_{3} \varphi$ be the gradient of $\varphi$ in all components, i.e. $\nabla_{3} \varphi=\left(\partial_{x} \varphi, \partial_{y} \varphi, \partial_{\gamma} \varphi\right)$.

Theorem 1. If

$$
\frac{\left|\nabla_{\boldsymbol{x}} \varphi\right|}{\left|\partial_{\gamma} \varphi\right|} \leq \frac{1}{3 w}
$$

\footnotetext{
${ }^{2}$ From (9), it is observed that the derivative of $\varphi$ with respect to $\gamma$ is zero everywhere except where $\varphi$ changes, i.e. $u(x)=\gamma$.
}

then constraint $\left|\nabla_{\boldsymbol{x}} u\right| \leq \frac{1}{3 w}$ is satisfied by any $u$ constructed by thresholding $\varphi$ at some value and applying (10). Proof: The gradient of $\nabla_{3} \varphi$ at point $(x, y, \gamma)$ is perpendicular to its level set surface at that point. That is, if we let $L_{\nu}$ to be the $\nu$-level surface of $\varphi$, let $v_{1}$ be tangent to $L_{\nu}$ at $(x, y, \gamma)$, and let $v_{2}=\nabla_{3} \varphi(x, y, \gamma)$, then $\left\langle v_{1}, v_{2}\right\rangle=0$.

Based on (9), $u$ is a level set of $\varphi$ no matter where $\varphi$ is thresholded. The standard technique for finding a vector tangent to the surface defined by $u$ is to choose a unit vector in $x$ and $y$ and set the $\gamma$ component equal to the rate of change of $u$ in the chosen direction. Specifically, this means

$$
v=\left(\frac{\partial_{x} u}{\left|\nabla_{\boldsymbol{x}} u\right|}, \frac{\partial_{y} u}{\left|\nabla_{\boldsymbol{x}} u\right|},\left|\nabla_{\boldsymbol{x}} u\right|\right)
$$

is tangent to the surface $u$, and thus is orthogonal to $\nabla_{3} \varphi$ at any point $(x, y, u(x, y))$. Thus

$$
\begin{aligned}
0 & =\left\langle v, \nabla_{3} \varphi\right\rangle \\
& =\frac{\left\langle\nabla_{\boldsymbol{x}} u, \nabla_{\boldsymbol{x}} \varphi\right\rangle}{\left|\nabla_{\boldsymbol{x}} u\right|}+\partial_{\gamma} \varphi\left|\nabla_{\boldsymbol{x}} u\right| \\
\left|\nabla_{\boldsymbol{x}} u\right| & =-\frac{\left\langle\nabla_{\boldsymbol{x}} u, \nabla_{\boldsymbol{x}} \varphi\right\rangle}{\left|\nabla_{\boldsymbol{x}} u\right|} \cdot \frac{1}{\partial_{\gamma} \varphi} \\
\left|\nabla_{\boldsymbol{x}} u\right| & \leq \frac{\left|\nabla_{\boldsymbol{x}} \varphi\right|}{\left|\partial_{\gamma} \varphi\right|} \leq \frac{1}{3 w},
\end{aligned}
$$

where the last step uses $\frac{\langle a, b\rangle}{|a|} \leq|b|$.

The objective now is to solve:

$$
\begin{gathered}
\underset{\varphi \in \mathcal{D}_{2}}{\arg \min } \int_{\Omega} \int_{\Gamma}\left|\nabla_{\boldsymbol{x}} \varphi\right|+\rho\left|\partial_{\gamma} \varphi\right| d \gamma d \boldsymbol{x}, \\
\mathcal{D}_{2}=\left\{\varphi \in \mathcal{D}_{1}|| \nabla_{\boldsymbol{x}} \varphi \mid \leq \frac{\left|\partial_{\gamma} \varphi\right|}{3 w}\right\} .
\end{gathered}
$$

\section{Optimization}

Due to the discontinuity in the Euler-Lagrange equation of [21], we use a primal-dual algorithm, [4, 5], to obtain the global solution. Defining the dual variable $\boldsymbol{p}=$ $\left(p_{1}, p_{2}, p_{3}\right)^{T}$, we can write the total variation part of 21] as

$$
\left|\nabla_{\boldsymbol{x}} \phi(\boldsymbol{x}, \gamma)\right|=\max _{\left|\boldsymbol{p}^{\prime}\right| \leq 1}\left\langle\boldsymbol{p}^{\prime}(\boldsymbol{x}, \gamma), \nabla_{\boldsymbol{x}} \phi(\boldsymbol{x}, \gamma)\right\rangle
$$

where $\boldsymbol{p}^{\prime}=\left(p_{1}, p_{2}\right)$. In 21], recalling that $-1 \leq \partial_{\gamma} \phi \leq 0$, it can be easily shown

$$
\rho(\boldsymbol{x}, \gamma)\left|\partial_{\gamma} \phi(\boldsymbol{x}, \gamma)\right|=\max _{-p_{3} \leq \rho} p_{3}(\boldsymbol{x}, \gamma) \partial_{\gamma} \varphi(\boldsymbol{x}, \gamma)
$$


Using these dual variables, the optimization problem of 21) becomes a min-max problem

$$
\begin{gathered}
\underset{\varphi \in \mathcal{D}_{2}}{\arg \min }\left\{\int _ { \Omega } \int _ { \Gamma } \left(\max _{\left|\boldsymbol{p}^{\prime}\right| \leq 1}\left\langle\boldsymbol{p}^{\prime}(\boldsymbol{x}, \gamma), \nabla_{\boldsymbol{x}} \phi(\boldsymbol{x}, \gamma)\right\rangle+\right.\right. \\
\left.\left.\max _{-p_{3} \leq \rho} p_{3}(\boldsymbol{x}, \gamma) \partial_{\gamma} \varphi(\boldsymbol{x}, \gamma)\right) d \gamma d \boldsymbol{x}\right\} \\
=\underset{\varphi \in \mathcal{D}_{2}}{\arg \min _{2}}\left\{\int_{\Omega} \int_{\Gamma} \max _{\boldsymbol{p} \in \mathcal{C}}\left\langle\nabla_{3} \varphi, \boldsymbol{p}\right\rangle\right\},
\end{gathered}
$$

Combining the constraints in 23] and 24] gives [20]

$$
\mathcal{C}=\mathcal{C}_{1}=\left\{\boldsymbol{p} \in \mathbb{R}^{3} \mid \sqrt{p_{1}^{2}+p_{2}^{2}} \leq 1, p_{3} \geq-\rho\right\},
$$

a cylinder with radius one, open on one end (cf. Fig. 4(a)). Recalling that $\partial_{\gamma} \varphi \leq 0, p_{3}$ that maximizes (26) is always as negative as possible, i.e. $p_{3}=-\rho$. Thus for $\mathcal{C}=\mathcal{C}_{1}$, the maximizing $\boldsymbol{p}$ is always on the circle at the base of the cylinder, highlighted in red in Fig. 4(a).

Unfortunately, while minimizing $\varphi$ over $\mathcal{D}_{1}$ can be done efficiently, the gradient magnitude constraint $\mathcal{D}_{2}$ imposes (in order to enforce the thickness constraint) makes the minimization of (26) for $\varphi$ difficult.

We will show that by moving the burden of enforcing the thickness constraint to the dual space, the optimization problem becomes much easier. Specifically, we will constrain $\varphi$ to $\mathcal{D}_{1}$, and introduce a new space for the dual variables, $\mathcal{C}_{2}$, satisfying the following properties:

1. $\mathcal{C}_{2}$ is convex;

2. if $\varphi$ satisfies $(15)$ then

$$
\max _{\boldsymbol{p} \in \mathcal{C}_{2}}\left\langle\nabla_{3} \varphi, \boldsymbol{p}\right\rangle=\max _{\boldsymbol{p} \in \mathcal{C}_{1}}\left\langle\nabla_{3} \varphi, \boldsymbol{p}\right\rangle ;
$$

3. if $\varphi$ does not satisfy (15) then (26) becomes arbitrarily large and that choice of $\varphi$ will be disallowed:

$$
\max _{\boldsymbol{p} \in \mathcal{C}_{2}}\left\langle\nabla_{3} \varphi, \boldsymbol{p}\right\rangle=\infty .
$$

Note that since 15 must be enforced at each spatial location, conditions 2 and 3 must also hold at each spatial location, thus the integrals are dropped from (26).

Theorem 2. A set that satisfies the three above conditions is the truncated cone defined by

$$
\mathcal{C}_{2}=\left\{\boldsymbol{p} \mid \sqrt{p_{1}^{2}+p_{2}^{2}} \leq 3 w\left(p_{3}+\rho\right)+1, p_{3} \geq-\rho\right\} .
$$

Proof. As a truncated cone, $\mathcal{C}_{2}$ is convex, so condition 1 is satisfied. To show 2 and 3 are satisfied, we will determine, for a given $\nabla_{3} \varphi$, the value of

$$
\max _{\boldsymbol{p} \in \mathcal{C}_{2}}\left\langle\nabla_{3} \varphi, \boldsymbol{p}\right\rangle .
$$

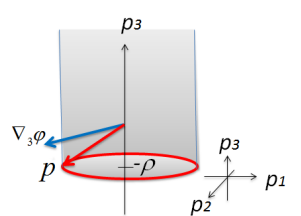

(a)

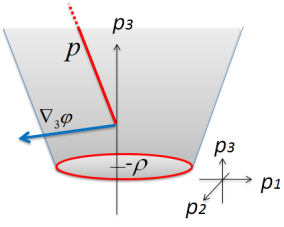

(b)

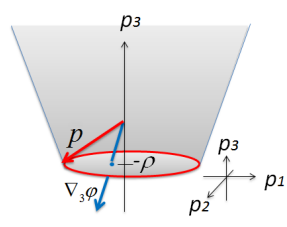

(c)
Figure 4: Valid sets for dual variables p. (a) Set $\mathcal{C}_{1}[20]$ (without any geometrical constraint). (b-c) Set $\mathcal{C}_{2}$; the truncated cone that impose our constraint (15). (b) When (15) is not satisfies $\mathbf{p}$ becomes $\infty$. (c) When 15 is satisfied we obtain the same solution as (a).

We first note that the circle at the base of the truncated cone is the same as the circle at the base of the cylinder defined by $\mathcal{C}_{1}$. Thus, if the $\boldsymbol{p}$ maximizing (31) lies on this circle (the red set in Fig. 4(c)), then (28) is satisfied. We now simplify this problem by reducing the possible forms for $\nabla_{3} \varphi$ and $\boldsymbol{p}$.

Since the condition (15) and $\mathcal{C}_{2}$ are rotationally symmetric with respect to the first two components, we can assume the second component of $\nabla_{3} \varphi$ is 0 , i.e. rotating $\nabla_{3} \varphi$ does not change (31). Further, if $\nabla_{3} \varphi$ is scaled by some constant, the maximizing $\boldsymbol{p}$ is not changed. Thus, we let $\nabla_{3} \varphi=(\ell, 0, a)$, where $\ell=\left|\nabla_{\boldsymbol{x}} \varphi\right|$ is held constant and $\partial_{\gamma} \varphi=a \leq 0$ is the only free parameter.

The maximizing $\boldsymbol{p}$ is always on the boundary of $\mathcal{C}_{2}$, since scaling $\boldsymbol{p}$ until it is on the boundary increases (31). Further, the first two components of the maximizing $p$ must align with the first two components of $\nabla_{3} \varphi$, so $p_{2}=0$.

If $\boldsymbol{p}$ is on the circle at the base of the cone, it would take the form $\boldsymbol{p}=(b, 0,-\rho)$, where $0 \leq b \leq 1$ is a free variable, and (31) becomes

$$
\max _{\mathbf{p}}\left\langle\boldsymbol{p}, \nabla_{3} \varphi\right\rangle=\max _{b}(b \ell-a \rho),
$$

which is maximized for $b=1$, i.e. a $p$ on the edge of the circle (the red set in Fig. 4(c)), so no maximizing $p$ is on the inner part of the circle.

If $\boldsymbol{p}$ is on the surface of the cone, it would take the form $\boldsymbol{p}=(3 w(b+\rho)+1,0, b)$ (from (30), where $b \geq-\rho$ is a free variable. This gives

$$
\begin{aligned}
\max _{\mathbf{p}}\left\langle\boldsymbol{p}, \nabla_{3} \varphi\right\rangle & =\max _{b}(\ell(3 w b+3 w \rho+1)+a b) \\
& =\max _{b}(b(3 w \ell+a)+3 w \rho \ell+\ell) .
\end{aligned}
$$

If $(3 w \ell+a) \leq 0$, (34) is maximized by minimizing $b$, i.e. $b=-\rho$. This corresponds to $\boldsymbol{p}$ on the edge of the circle (the red set in Fig. 4(c)), which implies (28) is satisfied. If $(3 w \ell+a)>0$, 34 is maximized by $b \rightarrow \infty$, and (31) gets arbitrarily large, satisfying 29] (cf. Fig. 4(b)).

To complete the proof, we note that $(3 w \ell+a) \leq 0$ is 
equivalent to the thickness constraint (15):

$$
\begin{aligned}
(3 w \ell+a) & \leq 0 \\
3 w\left|\nabla_{\boldsymbol{x}} \varphi\right| & \leq-\nabla_{\gamma} \varphi \\
\left|\nabla_{\boldsymbol{x}} \varphi\right| & \leq \frac{\left|\nabla_{\gamma} \varphi\right|}{3 w} .
\end{aligned}
$$

To find the optimal solution for $\varphi \in \mathcal{D}_{1}$ and $\boldsymbol{p} \in \mathcal{C}_{2}$, we perform the following primal and dual steps [3]:

Primal step Find the minimum $\varphi$ for a fixed $p$ :

$$
\varphi^{k+1}=\underset{\varphi \in \mathcal{D}_{1}}{\arg \min } \int_{\Omega \times \Gamma}\left\langle\nabla_{3} \varphi, \boldsymbol{p}^{k}\right\rangle+\frac{\left(\varphi-\varphi^{k}\right)^{2}}{2 t_{\varphi}} .
$$

Dual step Find the maximum $p$ for a fixed $\varphi$ :

$$
\boldsymbol{p}^{k+1}=\underset{\boldsymbol{p} \in \mathcal{C}_{2}}{\arg \max } \int_{\Omega \times \Gamma}\left\langle\nabla_{3} \varphi^{k+1}, \boldsymbol{p}\right\rangle+\frac{\left(\boldsymbol{p}-\boldsymbol{p}^{k}\right)^{2}}{2 t_{p}} .
$$

$t_{\varphi}$ and $t_{p}$ are the step sizes of the primal and dual update equations (here we used $t_{\varphi}=0.01$ and $t_{p}=5$ ). The solutions of (37) and (38) are derived from the EulerLagrange equations and projecting the obtained solutions to their valid sets (cf. Algorithm 1 in Appendix A).

After finding the global solution for the relaxed optimization problem, the labeling function $u$ is recovered by thresholding $\varphi$ at 0.5 and applying $(10)$. It can be proven that thresholded minimizers of the relaxed problem are the minimizers of the binary problem (13) (cf. Appendix B).

We note that our framework can be extended to multiple nested regions by dividing $\Gamma$ into more than 3 intervals in (1). The thickness constraint between consecutive regions can be set by adjusting $w$ and the interval length for each region in (1).

\section{Experiments and discussion}

In this section, we demonstrate advantages of our work over popular state-of-the-art segmentation methods and compare our framework with the analogous discrete work of Delong and Boykov (DB) [11].

\subsection{Synthetic data}

In our first experiment, we compare our method with DB in terms of memory usage and metrication error on a simple synthetic example.

Metrication error is defined as the artifacts which appear in graph-based segmentation methods due to penalizing region boundaries only across axis aligned edges. In Fig. 5, the goal is to segment the three-region object from the background. Fig. 5 (b-d) resulted from DB's method for 4,8 and 16 graph connectivity. Note the metrication artifacts in Fig. 5(b-c). Increasing the graph connectivity reduces metrication error, but also increases memory usage.

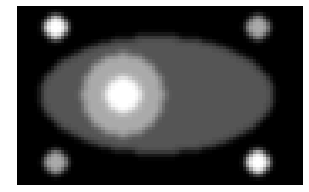

(a) Original image

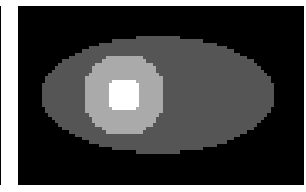

(b) GC: 4-connected

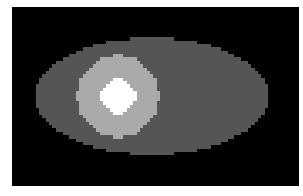

(c) GC: 8-connected

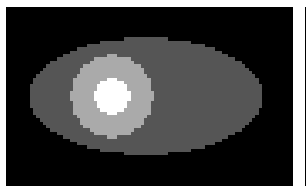

(d) GC: 16-connected

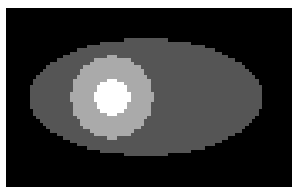

(e) Our result

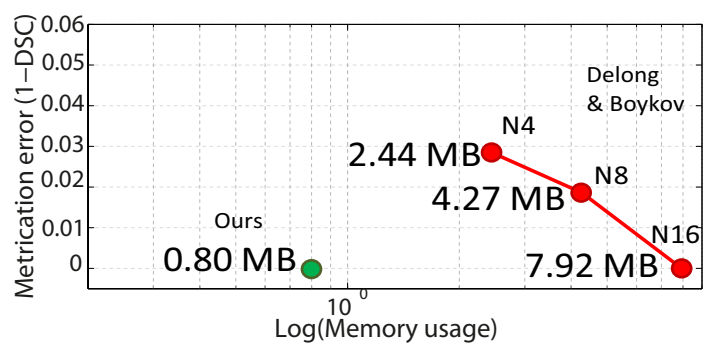

(f) Metrication error vs. memory usage

Figure 5: Synthetic three-region object+background segmentation. (b-d) DB graph cuts based method [11] with different connectivities. (e) Our segmentation results. (f) Metrication error vs. memory usage: red curve: GC-based method; green circle: our method.

Memory usage of our method and the graph-based methods is seen in Fig. 5(f). The red curve in Fig. 5(f) illustrates the metrication error (1 - Dice similarity coefficient(DSC)) vs. memory usage of [11] for 4, 8 and 16 connectivity, while the green circle represents our method. Here, removing the metrication error in the graph-based method requires 16 connectivity, even for these smooth objects. This requires $\sim 10$ times more memory than our method ( 0.80 vs. $7.92 \mathrm{MB})$.

\subsection{Microscopy/Histology cell segmentation}

We applied our method to a set of 20 different histology and microscopy images consist of multiple instances of multi-region cells. In these experiments, we show how containment and detachment with thickness constraints are useful for cell segmentation and compare our method with DB [11] in terms of memory usage and metrication error.

Fig. 6 shows a typical microscopy image with multiple cells, where nuclei are typically contained inside a cell membrane. Fig. 6(b) and (c) show the segmentation results with a thickness of $w=2$ pixels for DB (with 4connectivity) and our method, respectively. Metrication error can be clearly seen in Fig. 6(b). Our method also requires less memory (7.91 MB vs. $33.90 \mathrm{MB}$ ).

Changing the thickness of the containing region allows 


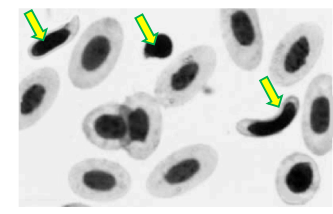

(a)

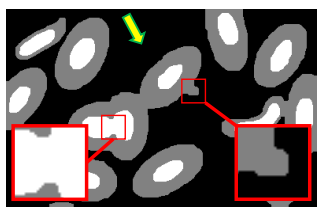

(b)

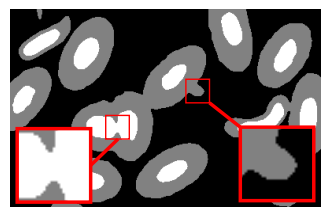

(c)

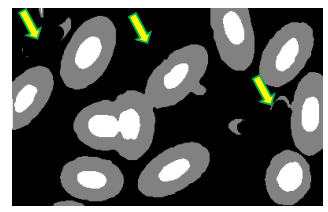

(d)

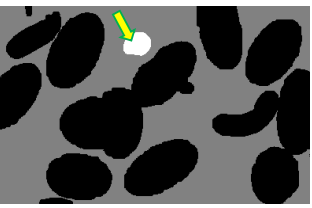

(e)

Figure 6: Cell segmentation in a microscopy imagery. (a) Original image, $250 \times 395$ pixels. Arrows show abnormal cells. (b) Result of [11], $33.90 \mathrm{MB}$. (c) Our result (thickness= 2 pixels, $7.91 \mathrm{MB}$ ) . (d) Our result to segment only normal looking (elliptical) cells (thisckness=10 pixels, $7.91 \mathrm{MB})$. Note that [11] needs $\sim 313.41 \mathrm{MB}$ extra memory (347.31 MB in total) to impose thickness of 10 pixels while the memory usage of our method is independent of thickness constraint. (e) Segmenting isolated nuclei by imposing detachment constraint (7).

Table 1: DSC and memory usage comparison (20 images).

\begin{tabular}{|c|c|c|}
\hline Method & DSC & Memory (MB) \\
\hline \hline No containment [20] & $0.6478 \pm 0.06$ & $8.9 \pm 7.2$ \\
\hline DB [11] & $0.9065 \pm 0.08$ & $180.0 \pm 204.7$ \\
\hline Ours & $0.9158 \pm 0.07$ & $8.9 \pm 7.2$ \\
\hline
\end{tabular}

us to control which objects are segmented. In Fig. 6a), to exclude the abnormal cells (arrows) from the segmentation, we increase the thickness of the outer region (membrane) from 2 to 10 pixels, resulting in Fig. 6(d). In DB, increasing the thickness requires more edges be added to the underlying graph, and increasing the thickness from 2 to 10 pixels requires an extra $\sim 313.41 \mathrm{MB}$ memory, an almost 10-fold increase. Thickness can be increased in our method by simply changing the value of $w$.

Fig. 6 (e) demonstrates the usage of a detachment constraint (cf. (7)), identifying nuclei that are not surrounded by a cell membrane.

Fig. 8 shows results for 7 other images, comparing segmentations generated using a continuous method without a containment constraint [20], DB with 4 connectivity, and our method. These results illustrate the importance of a containment constraint (first column) and also show the effects of metrication error (second column). Table 1 summarizes the mean accuracy and memory usage of the 3 methods across all 20 images, and Fig. 7 compares the memory usage vs. image size of our method and DB across all $20 \mathrm{im}-$ ages. We note that some of the images (e.g. the bottom two rows in Fig. (8) have 4 regions segmented, and Fig. 7 shows that the memory usage in graph-based methods tends to increase proportionally more than our continuous method in these cases.

On average, our method converges after 200 iterations for a $256 \times 256$ image. Using non-optimized MATLAB code on a standard $2.3 \mathrm{GHz}$ CPU with 6GB RAM, the graph cuts-based method [11] tends to run 2-3 times faster than our method but with more memory usage.

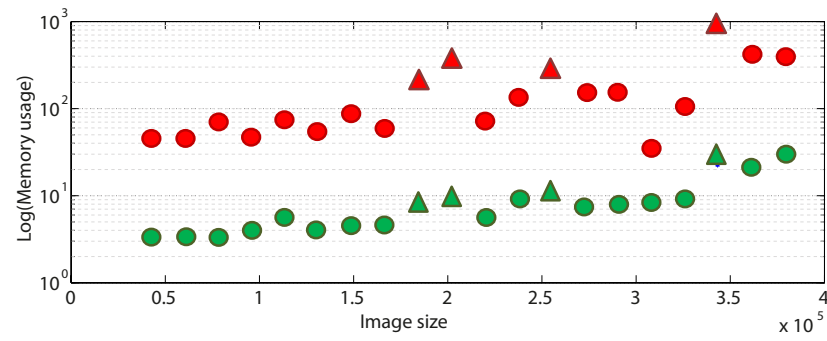

Figure 7: Memory efficiency: DB (in red) vs. ours (in green). $\bigcirc$ : 3-region segmentation; $\Delta$ : 4-region segmentation. Memory usage ratio (DB/ours) for 3 regions: $14.63 \pm 4.52$ and for 4 regions: $32.40 \pm 8.35$.

\section{Conclusion}

We introduced a variational framework to augment the conventional MS model for segmenting multi-region objects. We proposed a labeling function that allows us to enforce useful geometric constraints such as containment and detachment. By using this framework, a user can easily apply high level intuitive geometric constraints to improve segmentation results without the need for a deep understanding of how the method works. Our method compares favorably with analogous graph cuts-based methods in terms of metrication error and memory efficiency.

\section{References}

[1] X. Bresson, P. Vandergheynst, and J. Thiran. A variational model for object segmentation using boundary information and shape prior driven by the Mumford-Shah functional. IJCV , 68:145-162, 2006.

[2] E. S. Brown, T. F. Chan, and X. Bresson. Completely convex formulation of the Chan-Vese image segmentation model. IJCV, 98(1):103-121, 2012.

[3] A. Chambolle. An algorithm for total variation minimization and applications. JMIV, 20(1):89-97, 2004.

[4] A. Chambolle. Total variation minimization and a class of binary MRF models. In EMMCVPR, pages 136-152, 2005.

[5] T. Chan, G. Golub, and P. Mulet. A nonlinear primal-dual method for total variation-based image restoration. SIAM J. Sci. Comput., 20(6):1964-1977, 1999. 
[6] T. Chan, B. Sandberg, and L. Vese. Active contours without edges for vector-valued images. J. Visual Commun. Image Represent., 11(2):130-141, 2000.

[7] T. F. Chan, S. Esedoglu, and M. Nikolova. Algorithms for finding global minimizers of image segmentation and denoising models. SIAM J. Appl. Math., 66(5):1632-1648, 2006.

[8] G. Chung and L. A. Vese. Image segmentation using a multilayer level-set approach. Comput. Visual. Sci., 12(6):267$285,2009$.

[9] O. Colliot, O. Camara, and I. Bloch. Integration of fuzzy spatial relations in deformable models-application to brain MRI segmentation. Pattern Recognit., 39(8):1401-1414, 2006.

[10] T. Cootes, G. Edwards, and C. Taylor. Active appearance models. IEEE TPAMI, pages 484-498, 1998.

[11] A. Delong and Y. Boykov. Globally optimal segmentation of multi-region objects. In ICCV, pages 285-292, 2009.

[12] P. F. Felzenszwalb and O. Veksler. Tiered scene labeling with dynamic programming. In CVPR, pages 3097-3104, 2010.

[13] W. Fleming and R. Rishel. An integral formula for total gradient variation. Archiv der Mathematik, 11(1):218-222, 1960.

[14] S. Gould, J. Rodgers, D. Cohen, G. Elidan, and D. Koller. Multi-class segmentation with relative location prior. IJCV, 80(3):300-316, 2008.

[15] H. Ishikawa. Exact optimization for Markov random fields with convex priors. IEEE TPAMI, 25(10):1333-1336, 2003.

[16] K. Li, X. Wu, D. Chen, and M. Sonka. Optimal surface segmentation in volumetric images-a graph-theoretic approach. IEEE TPAMI, 28(1):119-134, 2006.

[17] A. Litvin and W. C. Karl. Coupled shape distribution-based segmentation of multiple objects. In IPMI, pages 345-356, 2005.

[18] D. Mumford and J. Shah. Optimal approximations by piecewise smooth functions and associated variational problems. Commun. Pure Appl. Math., 42(5):577-685, 1989.

[19] N. Paragios. A variational approach for the segmentation of the left ventricle in cardiac image analysis. IJCV, 50(3):345362, 2002.

[20] T. Pock, T. Schoenemann, G. Graber, H. Bischof, and D. Cremers. A convex formulation of continuous multi-label problems. In $E C C V$, pages 792-805. 2008.

[21] E. Strekalovskiy and D. Cremers. Generalized ordering constraints for multilabel optimization. pages 2619-2626, 2011.

[22] E. Strekalovskiy, C. Nieuwenhuis, and D. Cremers. Nonmetric priors for continuous multilabel optimization. In ECCV, pages 208-221. 2012.

[23] E. Ukwatta, J. Yuan, M. Rajchl, and A. Fenster. Efficient global optimization based 3D carotid AB-LIB MRI segmentation by simultaneously evolving coupled surfaces. In $M I C$ CAI, pages 377-384. 2012.

[24] J. Ulén, P. Strandmark, and F. Kahl. An efficient optimization framework for multi-region segmentation based on Lagrangian duality. IEEE TMI, 2012.

[25] A. Vazquez-Reina, E. Miller, and H. Pfister. Multiphase geometric couplings for the segmentation of neural processes. In CVPR, pages 2020-2027, 2009.
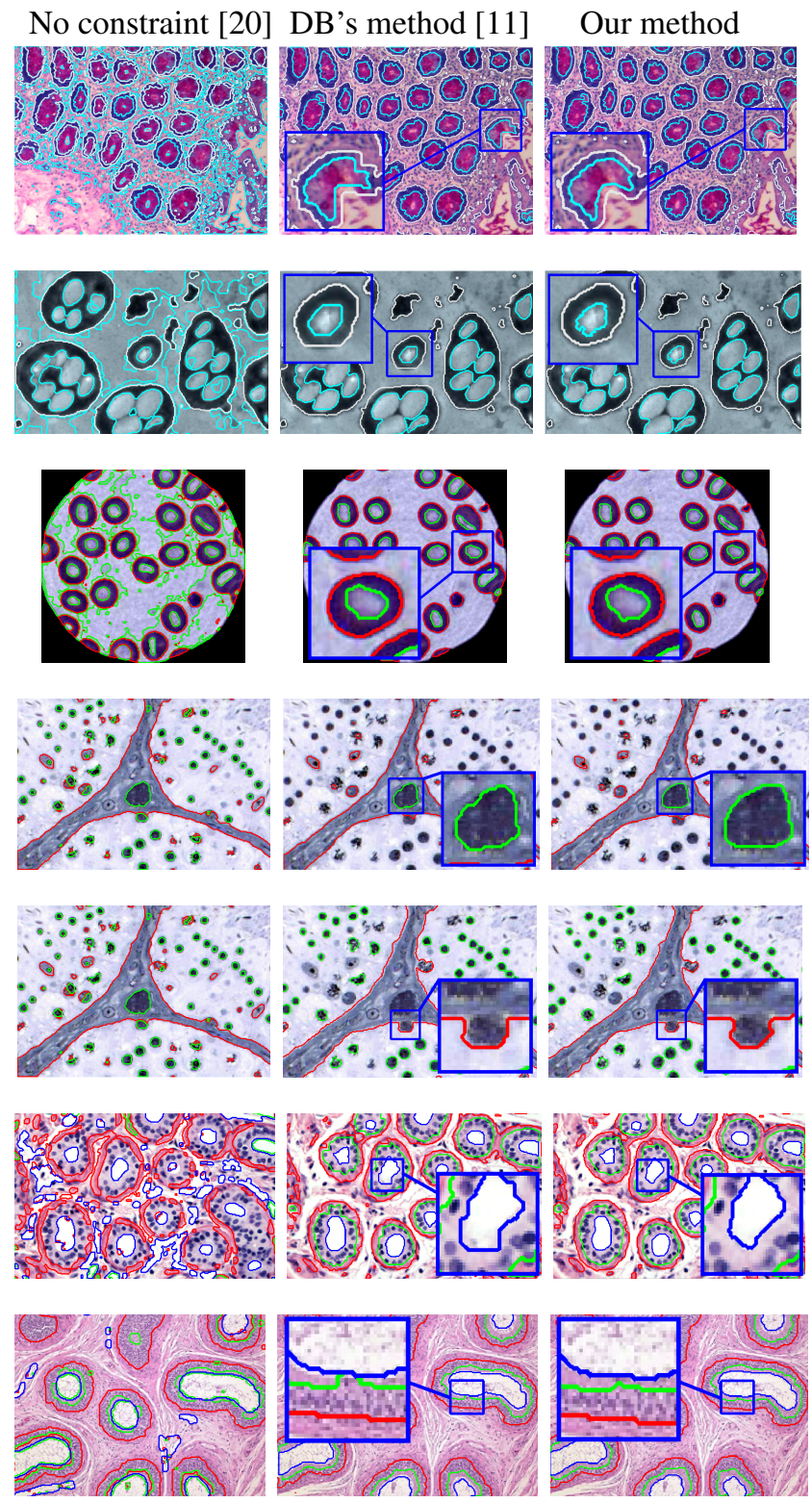

Figure 8: Incorporating geometrical constraint into the segmentation of histology and microscopy images.

[26] L. Vese and T. Chan. A multiphase level set framework for image segmentation using the Mumford and Shah model. IJCV, 50(3):271-293, 2002.

[27] Z. Wang and B. Vemuri. Tensor field segmentation using region based active contour model. In ECCV, pages 304315. 2004.

[28] X. Wu, X. Dou, A. Wahle, and M. Sonka. Region detection by minimizing intraclass variance with geometric constraints, global optimality, and efficient approximation. IEEE TMI, 30(3):814-827, 2011. 


\title{
Bounded Labeling Function for Global Segmentation of Multi-Part Objects with Geometric Constraints - ICCV 2013 paper supplementary material -
}

\author{
Masoud S. Nosrati, Shawn Andrews, Ghassan Hamarneh \\ Medical Image Analysis Lab, Simon Fraser University, BC, Canada \\ $\{$ smn6, sda56, hamarneh\}@sfu.ca
}

\section{Appendix A}

Algorithm 1 shows the alternating minimization scheme that is used to update $\varphi$ and $\boldsymbol{p}$ in (37) and (38).

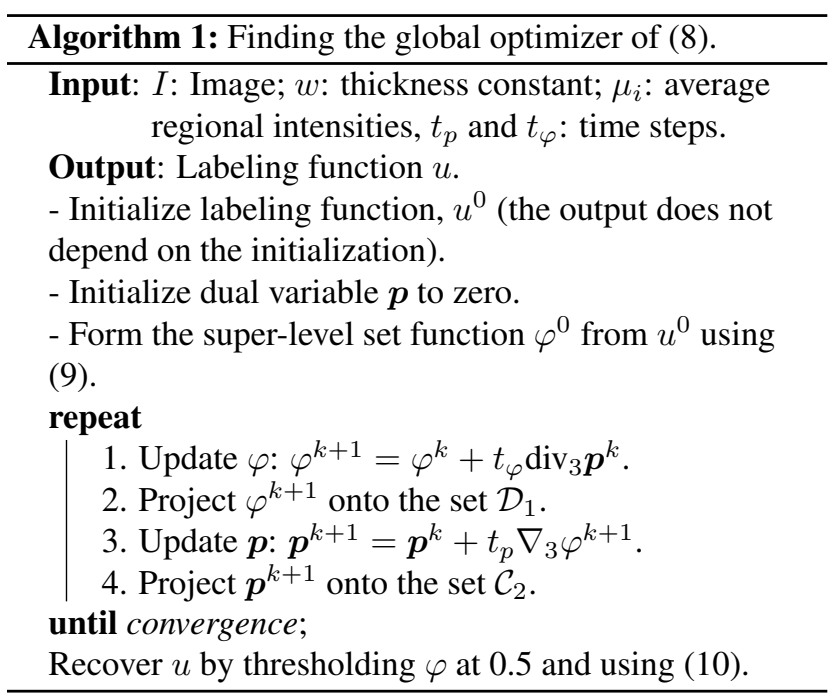

The update equations in lines 1 and 3 in Algorithm 1 are easily obtained by deriving the Euler-Lagrange equation for (37) and (38), respectively. We use Euclidean projector to reproject $\varphi^{k+1}$ and $\boldsymbol{p}^{k+1}$ onto the sets $\mathcal{D}_{1}$ and $\mathcal{C}_{2}$, respectively.

In our numerical implementation and discretization, assuming that $\Delta x$ and $\Delta y$ are spatial step sizes in $x$ and $y$ directions and $\Delta \gamma$ is the label discretization step, we approximate $(x, y, \gamma) \in \Omega \times \Gamma$ by $(i \Delta x, j \Delta y, k \Delta \gamma)$, where $(i, j, k)$ is the discrete location on the following Cartesian grid

$$
\{(i, j, k) \mid 1 \leq i \leq M, 1 \leq j \leq N, 1 \leq k \leq O\}
$$

and $M, N$ and $O$ denote the size of the grid. We use forward and backward finite differences to approximate $\nabla_{3} \varphi$ and $\operatorname{div}_{3} \boldsymbol{p}$, respectively:

$$
\begin{aligned}
& \left(\nabla_{3} \varphi\right)_{i, j, k}= \\
& \left(\frac{\varphi_{i+1, j, k}-\varphi_{i, j, k}}{\Delta x}, \frac{\varphi_{i, j+1, k}-\varphi_{i, j, k}}{\Delta y}, \frac{\varphi_{i, j, k+1}-\varphi_{i, j, k}}{\Delta \gamma}\right)^{T} \\
& \left(\operatorname{div}_{3} \boldsymbol{p}\right)_{i, j, k}= \\
& \frac{p_{i, j, k}^{1}-p_{i-1, j, k}^{1}}{\Delta x}+\frac{p_{i, j, k}^{2}-p_{i, j-1, k}^{2}}{\Delta y}+\frac{p_{i, j, k}^{3}-p_{i, j, k-1}^{3}}{\Delta \gamma}
\end{aligned}
$$

Here, we set $\Delta x=\Delta y=1$ and $\Delta \gamma=0.05$.

\section{Appendix B}

We follow Theorem 2 of Pock et al. [20] to prove that the thresholded minimizers of the relaxed problem $\left(\varphi \in \mathcal{D}_{1}\right)$ are the minimizers of the binary problem (13).

Theorem. Let $\varphi^{*} \in \mathcal{D}_{1}$ be the solution of the relaxed problem. Then for almost any threshold $\mu \in[0,1]$ the characteristics function $\mathbf{1}_{\left\{\varphi^{*} \geq \mu\right\}} \in\{0,1\}$ is also a minimizer of the binary variational problem (13).

Proof by contradiction. Since the relaxed problem is homogeneous of degree one, we make use of the generalized co-area formula to decompose it by means of the level sets of $\varphi$.

$$
\begin{aligned}
E(\varphi) & =\int_{\Omega} \int_{\boldsymbol{\Gamma}}|\nabla \varphi(\boldsymbol{x}, \gamma)|+\rho(\boldsymbol{x}, \gamma)\left|\partial_{\gamma} \varphi(\boldsymbol{x}, \gamma)\right| d \gamma d \boldsymbol{x} \\
& =\int_{0}^{1}\left(\int_{\Omega} \int_{\boldsymbol{\Gamma}}\left|\nabla \mathbf{1}_{\{\varphi \geq \mu\}}\right|+\rho(\boldsymbol{x}, \gamma)\left|\partial_{\gamma} \mathbf{1}_{\{\varphi \geq \mu\}}\right| d \gamma d \boldsymbol{x}\right) d \mu \\
& =\int_{0}^{1} E\left(\mathbf{1}_{\{\varphi \geq \mu\}}\right) d \mu .
\end{aligned}
$$

Assume to the contrary that $\mathbf{1}_{\left\{\varphi^{*} \geq \mu\right\}} \in\{0,1\}$ is not a global minimizer of the binary problem (13), i.e. there exists a binary function $\varphi^{\prime} \in\{0,1\}$ with $E\left(\varphi^{\prime}\right)<$ $E\left(\mathbf{1}_{\left\{\varphi^{*} \geq \mu\right\}}\right)$ for a set of $\mu \in[0,1]$. This directly implies that

$$
E\left(\varphi^{\prime}\right)=\int_{0}^{1} E\left(\varphi^{\prime}\right) d \mu<\int_{0}^{1} E\left(\varphi^{*} \geq \mu\right) d \mu=E\left(\varphi^{*}\right)
$$

which means that $\varphi^{*}$ is not a global minimizer of $E($.$) , con-$ tradicting our assumption. 